\title{
Uma avaliação acerca do perfil somatossensorial de indivíduos hemiparéticos
}

\author{
An evaluation of the somatosensory profile of hemiparetic individuals
}

\author{
R.S. Mota, T.M.A. Conceição, T.M.C. Soares, I. Lima e Silva, F.B. Cardoso, H. Beresford
}

\begin{abstract}
RESUMO
O objetivo do presente estudo foi avaliar o perfil somatossensorial de 18 indivíduos hemiparéticos espásticos vitimados de acidente vascular cerebral com e sem bloqueio visual. Como estratégia metodológica foram utilizados os testes de contração isométrica máxima para os músculos flexores e extensores do quadril, flexores e extensores do joelho e flexores plantares além de número de ciclos por minuto em bicicleta ergométrica, que foram realizados em dois instantes: olhos abertos e olhos fechados. Após análise estatística foi comprovado diferenças significativas entre os dois momentos para cada teste. Desta forma, pode-se concluir que parece haver falhas de comunicação entre os mecanismos neurais sensório-motores para a elaboração do ato motor voluntário dos indivíduos avaliados.

Palavras-chave: avaliação, somatossensorial, hemiparesia, AVC
\end{abstract}

The purpose of this study was to evaluate the somatosensory profile of 18 hemiparetic spastic victims of stroke with and without blocking vision. Maximal isometric contraction test was used for flexor and extensor muscles of the hip and knee, and flexor plantar muscles. The number of cycles per minute on stationary bike was also measured with eyes opened and closed. Significant differences were found suggesting the existence of miscommunication between sensory-motor neural mechanisms responsible for voluntary motor actions in these individuals.

Keywords: evaluation, somatosensory, hemiparesis, stroke

Submetido: 09.10.2009 | Aceite: 03.05.2010

Renata de Sousa Mota. Docente da Faculdade União Metropolitana de Educação e Cultura - Lauro de Freitas, Bahia, Brasil e do Instituto Baiano de Ensino Superior - Salvador, Bahia, Brasil.

Tatiana Maíta Alves Conceição. Docente do Centro Universitário Jorge Amado - Salvador, Bahia, Brasil.

Thais Miranda Curvelo Soares. Docente do Centro Universitário da Bahia - Salvador, Bahia, Brasil.

Iris Lima e Silva e Fabrício Bruno Cardoso. Pesquisador do LABFILC - Laboratório de Temas Filosóficos em Conhecimento Aplicado da Universidade Castelo Branco, Rio de Janeiro, Brasil.

Heron Beresford. Professor Adjunto da Universidade do Estado do Rio de Janeiro, Brasil - UERJ. Coordenador de Pesquisas no LABFILC - Laboratório de Temas Filosóficos em Conhecimento Aplicado, Rio de Janeiro, Brasil.

Endereço para correspondência: Renata de Sousa Mota, Rua Maranhão, 373/604, Pituba, Salvador, CEP: 41830 260, Bahia, Brasil.

E-mail: natamota@hotmail.com 
De acordo com a Organização Mundial de Saúde (OMS), descrito pelo Ministério da Saúde do Brasil (2004), o acidente vascular cerebral (AVC) é definido como uma síndrome clínica com desenvolvimento rápido de sinais clínicos de perturbação focal ou global da função cerebral, com possível origem vascular e com mais de 24 horas de duração.

De acordo com Teixeira-Salmela, Olney e Nadeau (1999), esses sinais clínicos estão relacionados com a localização e o tamanho da lesão, a natureza e as funções da área atingida e com a disponibilidade de um fluxo colateral no encéfalo e podem desencadear uma variedade de alterações no sistema somatossensorial, cujo funcionamento é responsável por transmitir e analisar o tato ou informações táteis de localizações internas e externas no corpo e na cabeça, sendo o produto final desse processo denominado de apreciação de sensações somáticas.

Segundo trabalhos realizados, seu comprometimento gera perturbações nas funções sensório-motoras, cognitivas, e ações que envolvem a linguagem e percepção intrasensorial, tornando estes indivíduos portadores de uma inabilidade funcional (Danckert, Saoud, \& Maruff, 2004; Sharp \& Brouwer, 1997; Teixeira-Salmela, Lima, Morais, \& Goulart, 2005; Teixeira-Salmela, Lima, Souza, \& Goulart, 2006).

Sendo assim, Franzoi e Kagohara (2007), Lent (2008) e Shumway e Woollacott (2003) postularam que as consequências da lesão em regiões responsáveis pelo processamento das informações sensório-motoras desencadeiam incapacidades variáveis a depender da sua extensão, caracterizadas por modificações no controle motor voluntário, com paralisia ou paresia do lado contra lateral à lesão (hemiplegia ou hemiparesia), alteração na marcha, espasticidade e hiperreflexia profunda, mudança na imagem corporal e agnosias.

Estudos anteriormente publicados indicam que o controle postural está diretamente relacionado com o funcionamento do sistema visual, do sistema vestibular e do sistema somatossensorial, assim como, a interação entre eles e a posterior relação com o planejamento motor e assim, com a execução do ato motor voluntário (Carello, Silva, Kinsella-Shaw, \& Turvey, 2008; Hortobágyi et al., 2009; Lent, 2008; Mochizuki \& Amadio, 2006; Shumway \& Woollacott, 2003). Esses sistemas oferecem subsídios adequados para a elaboração do movimento através dos feedbacks sensoriais, tornando o sistema motor cerebral dependente do sistema sensorial (Kandell, 2008; Lent, 2008; Magill, 2000; Silva et al., 2008).

Sendo assim, Franzoi e Kagohara (2007) e Mochizuki e Amadio (2006) relataram que em indivíduos portadores de hemiparesia após um AVC há alterações nestes órgãos e nos seus respectivos mecanismos e como conseqüência, eles não conseguem manter um equilíbrio na postura ereta e expressam modificação no desempenho da marcha.

Portanto, de acordo com Cardoso (2007), essas modificações na integração somatossensorial e suas repercussões na estruturação da imagem corporal e do seu movimento geram mudanças no sentido neurofilosófico do que é entendido sobre todas as dimensões do corpo humano.

Segundo Merleau-Ponty (1999), o ser humano consiste no centro da discussão sobre o conhecimento. Este nasce e faz-se sensível em sua corporeidade - "eu sou o meu corpo". Para ele, o corpo é o sentido de toda a existência humana. Desta forma, alterar a percepção da imagem de seu próprio corpo como acontece nos indivíduos incapacitados após sofrer um AVC, é modificar a consciência de toda a plenitude deste modelo com a sua percepção do mundo, tal como afirmado por Beresford (2006).

Diante da problemática referida, este estudo teve por objetivo avaliar o perfil somatossensorial de indivíduos hemiparéticos espásticos vitimados de AVC com e sem bloqueio visual. 


\section{MÉTODO}

Este estudo foi operacionalizado utilizando a estratégia metodológica de uma avaliação de contexto através de uma pesquisa experimental. Segundo Stufflebeam (1977), esta metodologia é o tipo mais básico de avaliação, cuja finalidade é a de fornecer um fundamento lógico para a determinação de objetivo. Especificamente, a referida avaliação define o meio ambiental concernente, descreve as condições reais e desejadas em relação ao referido ambiente, identifica as carências não supridas e fornece informações para se planejar ações necessárias para a resolução dos mesmos, como também as oportunidades não utilizadas e diagnostica os problemas que impedem o entendimento das necessidades de serem supridas e as oportunidades de serem utilizadas.

\section{Amostra}

A amostra deste estudo foi composta por 18 indivíduos hemiparéticos espásticos vitimados de AVC, de ambos os sexos, com idades entre 45 e 67 anos, que faziam fisioterapia em uma clínica escola da cidade de Salvador, Bahia Brasil, lúcidos e orientados, capazes de entender e executar comandos do avaliador e que apresentavam marcha independente sem o uso de qualquer dispositivo. Estes formavam um grupo denominado voluntário de acordo com a tecnologia da amostragem de Cochran (1956).

\section{Instrumentos e Procedimentos}

Para a concretização desta pesquisa, durante o mês de Março de 2009, os participantes do estudo foram submetidos aos seguintes testes, baseados nos trabalhos de Mochizuki e Amadio (2006) e Teixeira-Salmela et al. (1999, 2005, 2006), a saber:

Contração Muscular Máxima: Através de isometria sustentada com olhos abertos e posteriormente com olhos fechados com uso de venda da cor preta para oclusão da visão.Foi verificado o tempo que o indivíduo conseguia manter a contração isométrica máxima bilateral e simultânea dos seguintes grupamentos musculares, através de cronómetro portátil da marca Casio Lap Memory.

Extensores do quadril: Com o indivíduo deitado de decúbito dorsal sobre uma maca e membros inferiores em flexão, com apoio sobre a superfície plantar foi solicitado realizar o movimento da ponte e sustentá-la o máximo de tempo possível.

Flexores de quadril: Com o indivíduo em decúbito dorsal sobre uma maca, foi solicitado ao participante realizar flexão de quadril de ambas as pernas com joelhos extendidos simultaneamente até $45^{\circ}$ de amplitude articular e sustentá-las o máximo de tempo possível.

Extensores de joelhos: Com o indivíduo sentado sobre uma maca com membros inferiores pendentes, foi solicitado que o mesmo realizasse extensão dos dois joelhos de forma simultânea até $180^{\circ}$ de amplitude articular e sustentá-los o máximo de tempo possível.

Flexores de joelhos: Com o indivíduo deitado de decúbito ventral sobre uma maca, foi solicitado flexão dos dois joelhos simultaneamente até $90^{\circ}$ de amplitude articular e sustentação o máximo de tempo possível.

Plantiflexão: Com o indivíduo na postura de pé, de frente a uma parede lisa, com as mãos segurando esta e os pés à largura dos ombros foi solicitado que o mesmo permanecesse o maior tempo possível na ponta dos pés, realizando a flexão plantar.

Repetição de ciclos em bicicleta ergométrica vertical: Verificou-se o número de ciclos realizados num intervalo de tempo de 1 minuto em uma bicicleta ergométrica da marca Moviment, modelo 2700, com a carga mínima oferecida pela mesma em dois momentos, primeiramente com olhos abertos e depois o mesmo procedimento com olhos fechados.

Para a execução dos testes os participantes foram instruídos a estarem usando roupa confortável e tênis como calçado. 
Foi aferida a pressão arterial e a temperatura corporal para garantir integridade clínica.

O projeto desta pesquisa foi submetido ao Comitê de Ética em Pesquisa envolvendo seres humanos da Universidade Castelo Branco (UCB/RJ) e aprovada sob protocolo $\mathrm{n}^{\circ}$ 0163/2008.

A coleta de dados se deu conforme a resolução 196/96 do Conselho Nacional de Saúde do Ministério da Saúde Brasileiro. Assim, inicialmente foi solicitada a que todos os participantes assinassem o termo de consentimento livre e esclarecido.

\section{Análise estatística}

A análise dos dados deste estudo foi realizada com base na comparação de resultados estatísticos, utilizando-se o programa BIOESTAT 5.0.

Os resultados obtidos nas avaliações de contração muscular máxima de olhos abertos e olhos fechados foram calculados através da estatística descritiva, a média e o desvio padrão. Todos os dados foram considerados paramétricos pelo teste de Shapiro-Wilk, portanto, para uma comparação entre olhos abertos e olhos fechados utilizaram-se testes próprios para duas amostras pareadas já que cada participante deste estudo contribuiu com dois escores em cada teste.

Desta forma, a média das diferenças entre os instantes olhos abertos e olhos fechados foi analisada de forma paramétrica utilizando-se, para o efeito, $\mathrm{o}$ teste $\mathrm{t}$ de Student para amostras pareadas.

\section{RESULTADOS}

Na Tabela 1 são apresentados os dados dos resultados dos indivíduos avaliados em relação a sua capacidade de executar ciclos em uma bicicleta ergométrica durante 1 minuto, de olhos abertos e olhos fechados.

No instante de olhos abertos, os indivíduos apresentaram uma variação de resultados entre 35 e 84 ciclos e a média do grupo ficou em 58.44 ciclos por minuto, já no instante de olhos fechados a variação ficou entre 31 e 86 ciclos, e a média em 51.23 ciclos por minuto. Assim observa-se uma diferença entre as médias de 7.21 pontos, que se apresenta significante através do teste $\mathrm{t}(p=.02)$, comprovando assim a diferença entre os dois instantes.

Tabela 1

Avaliação da capacidade de reproduzir ciclos de bicicleta por 1 minuto

\begin{tabular}{lcc}
\hline & \multicolumn{2}{c}{ Bicicleta ergométrica } \\
\cline { 2 - 3 } & $\begin{array}{c}\text { Olhos } \\
\text { abertos }\end{array}$ & $\begin{array}{c}\text { Olhos } \\
\text { fechados }\end{array}$ \\
\hline Mínimo & 35.00 & 31.00 \\
Máximo & 84.00 & 86.00 \\
Amplitude Total & 49.00 & 55.00 \\
Mediana & 56.50 & 48.50 \\
Primeiro Quartil (25\%) & 49.25 & 41.25 \\
Média Aritmética & 58.44 & 51.23 \\
Desvio Padrão & 14.17 & 14.94 \\
Coeficiente de Variação & $24.25 \%$ & $29.14 \%$ \\
\hline
\end{tabular}

Já na Tabela 2, apresentam-se as médias dos testes que envolvem os movimentos do joelho. Para a sua extensão, a variação de resultados no instantes de olhos abertos apresentada pelos indivíduos ficou entre 3.75 e 45.00 segundos, sendo a média do grupo de 20.44 segundos.

Já no instante de olhos fechados a variação de resultados obtidos ficou entre 2.13 e 32.63 segundos, obtendo-se uma média igual a 15.26, o que faz perceber que existe uma diferença entre as médias dos instantes olhos abertos e olhos fechados de 5.18 segundos, diferença esta considerada significante, através do teste $\mathrm{t}(p=.03)$.

Ainda na Tabela 2 é possível perceber que os resultados relacionados à flexão de joelho nos instante de olhos abertos apresentaram uma variação entre .23 e 14.18 segundos, e uma média de 4.39 segundos, e no instante de olhos fechados a variação de resultados em relação à capacidade de flexão do joelho ficou entre .23 e 8.51 segundos e a média foi apenas 
de 2.77 segundos. Nota-se, então, que existe uma diferença de 1.62 segundos entre as médias dos dois instantes, diferença esta que foi significante estatisticamente $(p=.01)$.

A partir da Tabela 3 é possível observar o desempenho dos indivíduos em relação à capacidade de contração isométrica do quadril quanto à extensão e flexão, nos instantes de olhos abertos e olhos fechados. Em relação à extensão do quadril no instante de olhos abertos, a variação entre os resultados foi de 2.65 a 49.40 segundos, e uma média de 26.41 segundos.

No instante de olhos fechados a média foi de 3.47 segundos, com uma variação de resultados entre 1.00 e 7.57 segundos. Observa-se, então, uma melhor capacidade dos indivíduos em realizar a contração isométrica máxima do quadril em extensão de olhos abertos, já que foi obtida uma diferença entre as duas médias de 22.94 segundos, a qual é significativa a $p=.01$.

Já em relação à contração isométrica de flexão de quadril no instante de olhos abertos, a variação de resultados apresentados ficou entre 1.00 e 8.02 segundos e a média foi de 4.70 segundos.

No instante de olhos fechados a média foi de 3.47 segundos, com uma variação de resultados entre 1.00 e 7.57 segundos. Notase, então, uma diferença de 1.23 segundos, a qual também revelou uma expressão estatisticamente significativa $(p=.05)$.

Tabela 2

Avaliação acerca da contração isométrica máxima em extensão e flexão do joelho (tempo de contração em segundos)

\begin{tabular}{lcccc}
\hline & \multicolumn{2}{c}{$\begin{array}{c}\text { Contração isométrica máxima } \\
\text { extensão do joelho }\end{array}$} & \multicolumn{2}{c}{$\begin{array}{c}\text { Contração isométrica máxima } \\
\text { flexão do joelho }\end{array}$} \\
\cline { 2 - 5 } & Olhos abertos & Olhos fechados & Olhos abertos & Olhos fechados \\
\hline Mínimo & 3.75 & 2.13 & .23 & .23 \\
Máximo & 45.00 & 32.63 & 14.18 & 8.51 \\
Amplitude Total & 41.25 & 30.50 & 13.95 & 8.28 \\
Mediana & 16.68 & 12.76 & 4.15 & 2.79 \\
Primeiro Quartil (25\%) & 5.95 & 4.56 & 2.63 & 1.36 \\
Média Aritmética & 20.44 & 15.26 & 4.39 & 2.77 \\
Variância & 251.48 & 125.80 & 10.60 & 4.46 \\
Desvio Padrão & 15.86 & 11.22 & 3.26 & 2.11 \\
Coeficiente de Variação & $77.60 \%$ & $73.49 \%$ & $74.13 \%$ & $76.24 \%$ \\
\hline
\end{tabular}

Tabela 3

Avaliação acerca da contração isométrica máxima em extensão e flexão do quadril (tempo de contração em segundos

\begin{tabular}{lcccc}
\hline & \multicolumn{2}{c}{$\begin{array}{c}\text { Contração isométrica máxima } \\
\text { extensão do quadril }\end{array}$} & $\begin{array}{c}\text { Contração isométrica máxima } \\
\text { flexão do quadril }\end{array}$ \\
\cline { 2 - 5 } & Olhos abertos & Olhos fechados & Olhos abertos & Olhos fechados \\
\hline Mínimo & 2.65 & 1.00 & 1.00 & 1.00 \\
Máximo & 49.40 & 7.57 & 8.02 & 7.57 \\
Amplitude Total & 46.75 & 6.57 & 7.02 & 6.57 \\
Mediana & 23.38 & 2.98 & 4.27 & 2.98 \\
Primeiro Quartil (25\%) & 11.16 & 1.92 & 2.57 & 1.98 \\
Média Aritmética & 26.41 & 3.47 & 4.70 & 3.47 \\
Variância & 263.45 & 4.02 & 5.80 & 4.02 \\
Desvio Padrão & 16.23 & 2.01 & 2.41 & 2.01 \\
Coeficiente de Variação & $61.46 \%$ & $57.79 \%$ & $51.31 \%$ & $57.79 \%$ \\
\hline
\end{tabular}


Em relação à flexão plantar no instante de olhos abertos, os resultados dos indivíduos demonstraram uma variação entre 1.30 e 14.50 segundos com uma média de 6.77 segundos.

Já no instante de olhos fechados os resultados variaram entre .22 e 9.87 segundos e a média ficou em 3.56 segundos. A comprovação estatística desta diferença entre as médias foi feita através do teste $\mathrm{t}$ para duas amostras pareadas que revelou um $p=.04$.

\section{DISCUSSÃO}

Shumway e Woollacott (2003) definem o controle motor como a integração de sistemas orgânicos com o objetivo de gerir o movimento. Sendo assim, a partir dos resultados apresentados anteriormente pode-se sugerir que os indivíduos avaliados apresentam uma dificuldade em desenvolver o "modelo de processamento de informação" que envolve a captação do estímulo, a decodificação, a percepção, o processamento central, a decisão da resposta, bem como o seu planejamento e a sua efetivação para cumprir uma determinada tarefa motora quando no instante de olhos fechados (Lent, 2008; Magill, 2000; Silva et al., 2008). Ou seja, esses indivíduos apresentavam dificuldade em executar o movimento propriamente dito que é definido por Beresford (2006) como o comportamento motor dotado de significação ou com valor por ser oriundo de uma consciência intencional surgida de um problema, ou de uma carência.

Considera-se então possível que estes participantes apresentem uma dificuldade de conexão intersensorial entre o processamento das informações secundárias ou de associação e as informações terciárias ou de integração onde ocorrem a adição e combinação de todos os aspectos do estímulo recebido. Com isso, baseado nas afirmações de Magill (2000) e de Kandell (2008) e no estudo de Silva et al. (2008), os indivíduos avaliados podem ter uma baixa frequência de condução neural nos processos cognitivos que envolvem planejamento (córtex pré-frontal), organização da sequência (pré-motor) e envio das ações específicas dos movimentos a serem executados (córtex motor). Assim, isto parece afetar o envio de mensagens para dosagem de força e agilidade, alterando o que é fornecido pelos feedbacks sensoriais.

Sendo assim, os indivíduos podem ter apresentado problemas para organização da atividade consciente e a sua regulação, programação e verificação no instante de olhos fechados, o que os tornaram incompreensíveis em relação a tarefas motoras seguidas e simultâneas que foram executadas num determinado período de tempo e que exigem a atividade conjunta de vários grupos musculares (Carello et al., 2008; De Paula, 2007). Ou seja, os executantes não conseguiram pensar sobre os aspectos cognitivos, simbólicos ou processuais da tarefa motora realizada de olhos fechados da mesma forma que fizeram de olhos abertos, ou melhor, na ausência de movimento observável. Portanto, enquanto os julgamentos (ou expectativas) de eficácia pessoal diriam respeito à convicção que o sujeito tinha de poder executar com sucesso um comportamento necessário para produzir determinado resultado, as expectativas de resultado diriam respeito à estimativa de uma pessoa de que um dado comportamento iria conduzir a certos resultados (Cardoso, 2007; Danckert et al., 2004; Hortobágyi et al., 2009).

Por fim, considera-se provável que os indivíduos avaliados neste estudo apresentaram falhas de comunicação acerca das informações resultantes da análise perceptiva que prosseguem na ordem do processamento, fluindo a seguir para o estágio de decisão e depois o de programação de resposta, apresentando uma desorganização na resposta desejada, enviando assim para a musculatura prevista uma informação não correta em relação ao tempo de execução do movimento.

\section{REFERÊNCIAS}

Beresford, H. (2006). Documento de apoio à disciplina estatuto epistemológico da motricidade humana. Mestrado em Ciência da Motricidade Humana, Universidade Castelo Branco. Rio de Janeiro. 
Cardoso, F. (2007). A utilização do programa de potencialização cerebral para a melhoria no lançamento da bola de boliche para atletas da seleção juvenil colombiana $B$. Dissertação de mestrado não publicada, Universidade Castelo Branco, Rio de Janeiro, Brasil.

Carello, C., Silva, P. L., Kinsella-Shaw, J. M., \& Turvey, M. T. (2008). Muscle-based perception: Theory, research and implications for rehabilitation. Revista Brasileira de Fisioterapia, 12(5), 339-50.

Cochran, G. (1956). Tecnologia da amostragem. Rio de Janeiro: Editora Fundo de Cultura.

Danckert, J., Saoud, M., \& Maruff, P. (2004). Attention, motor control and motor imagery in schizophrenia: Implications for the role of the parietal cortex. Schizophrenia Research, 70(2), 241-261.

De Paula, F. V. (2007). Teoria de programação motora: Uma perspectiva da sua evolução teórica. Fisioterapia em Movimento, 20(2), 63-71.

Franzoi, A. C., \& Kagohara, N. S. (2007). Correlação do perfil de deambulação e velocidade da marcha em um grupo de pacientes hemiplégicos atendidos em um centro de reabilitação. Revista Acta Fisiátrica, 14(2), 78-81.

Hortobágyi, T., Solnik, S., Gruber, A., Rider, P., Steinweg, K., Helseth, J., et al. (2009). Interaction between age and gait velocity in the amplitude and timing of antagonist muscle coactivation. Gait \& Posture, 29, 558-564.

Kandell, E. (2008). Principles of neural science (4a ed.). New York: McGraw-Hill.

Lent, R. (2008). Neurociência da mente e do comportamento. Rio de Janeiro: Guanabara Koogan.

Magill, R. A. (2000). Aprendizagem motora: Conceitose aplicações. São Paulo: Edgard Blücher.

Merleau-Ponty, M. (1999). Fenomenologia da percepção. São Paulo: Martins Fontes.

Ministério da Saúde Brasil - Brasil (2004) Painel de indicadores do Sistema Único de Saúde (SUS) Consultado em 12 de Novembro de 2008 a partir de http://www.saude.gov.br.
Mochizuki, L., \& Amadio, A. C. (2006). As informações sensoriais para o controle postural. Fisioterapia em Movimento, 19(2), 1118.

Sharp, S. A., \& Brower, B. J. (1997). Isokinetic strength training of hemiparetic knee: Effects on function and spasticity. Archives of Physical Medicine and Rehabilitation, 78, 1231-1236.

Shumway, A. C., \& Woollacott, M. H. (2003). Controle motor: Teoria e aplicações práticas (2a ed.). Barueri: Manole.

Silva, V. F, Poly, M. W. O., Ribeiro, S. M. S., Calomeni, M. R., Pinto, M. V. M., \& Silva, A. L. S. (2008). Efeito agudo da estimulação cerebral, através de luz e som, no tempo de reação motora de jovens atletas. Lecturas: Educación Física y Deportes, 120(13). Consultado em 12 de Novembro de 2008 a partir de http://www.efdeportes.com/efd120/tempo-dereacao-motora-de-jovens-atletas.htm.

Stufflebeam, D. L. (1977, Abril). Overview of the joint committee's project on evaluation standards. Joint session of the American Educational Research Association and the National Council on Measurement in Education, New York, USA.

Teixeira-Salmela, L. F., Lima, R. C. M., Souza, A. C., \& Goulart, F. V. P. (2006). Mudança na qualidade de vida associadas ao treinamento de hemiplégicos em academias. Revista Fisioterapia em Movimento, 19(3), 75-82.

Teixeira-Salmela, L. F., Olney, S. J., \& Nadeau, S. (1999). Muscle strengthening and physical conditioning to reduce impairment and disability in chronic stroke survivors. Archives of Physical Medicine and Rehabilitation, 80, 12111218.

Teixeira-Salmela, L. F., Lima, R. C. M, Morais, S.G., \& Goulart, F. V. P. (2005). Assimetria e desempenho funcional em hemiplégicos crônicos antes e após programa de treinamento em academia. Revista Brasileira de Fisioterapia, 9(2), 227-233. 